\title{
Neglected Odontoid Fracture During Growth
}

\author{
Joon Sung Kim, MD, PhD, Bo Young Hong, MD, PhD, Seong Hoon Lim, MD, PhD \\ Department of Rehabilitation Medicine, St Vincent's Hospital, \\ College of Medicine, The Catholic University of Korea, Seoul, Korea
}

An 18-year-old female visited a rehabilitation clinic because of weakness of both hands and gait disturbance for $>8$ years. She showed poor balance and weakness of the whole limb, with the right side more severely involved. In the medical interview, she did not remember the exact starting point of the weakness or any history of trauma. She recalled recognizing the different sizes of her hands at the age of 11 years. After this, her right-hand weakness and gait disturbance progressed. Laboratory examination for systemic disease, routine complete blood count, blood chemistry with electrolytes and muscle enzymes, and autoantibodies revealed no abnormal findings. Other tests such as brain magnetic resonance imaging (MRI) did reveal other hidden diseases or congenital anomalies. Autonomic dysfunction developed, and paresthesia of both hands caused difficulty of activities in daily living. Brain computed tomography did not reveal any abnormality. Plain radiography of the cervical spine revealed the old fracture of the odontoid process (Fig. 1A). MRI of the cervical spine revealed the old fracture of the odon- toid process and myelopathy at the $\mathrm{C} 1 / \mathrm{C} 2$ levels (Fig. 1B). She underwent spine surgery with "open reduction, C1-2 with temporary lateral mass screw fixation, $\mathrm{Cl}$, right". Thereafter, her neurological progression ceased. Three years after the operation, neurological deterioration did not occur or progress, except for the remaining gait disturbance, autonomic dysfunction, and paresthesia.

\section{CONFLICT OF INTEREST}

No potential conflict of interest relevant to this article was reported.

\section{AUTHOR CONTRIBUTION}

Conceptualization: Lim SH. Methodology: Kim JS, Hong BY, Lim SH. Visualization: Kim JS, Hong BY, Lim SH. Writing - review and editing: Lim SH. Approval of final manuscript: all authors. 

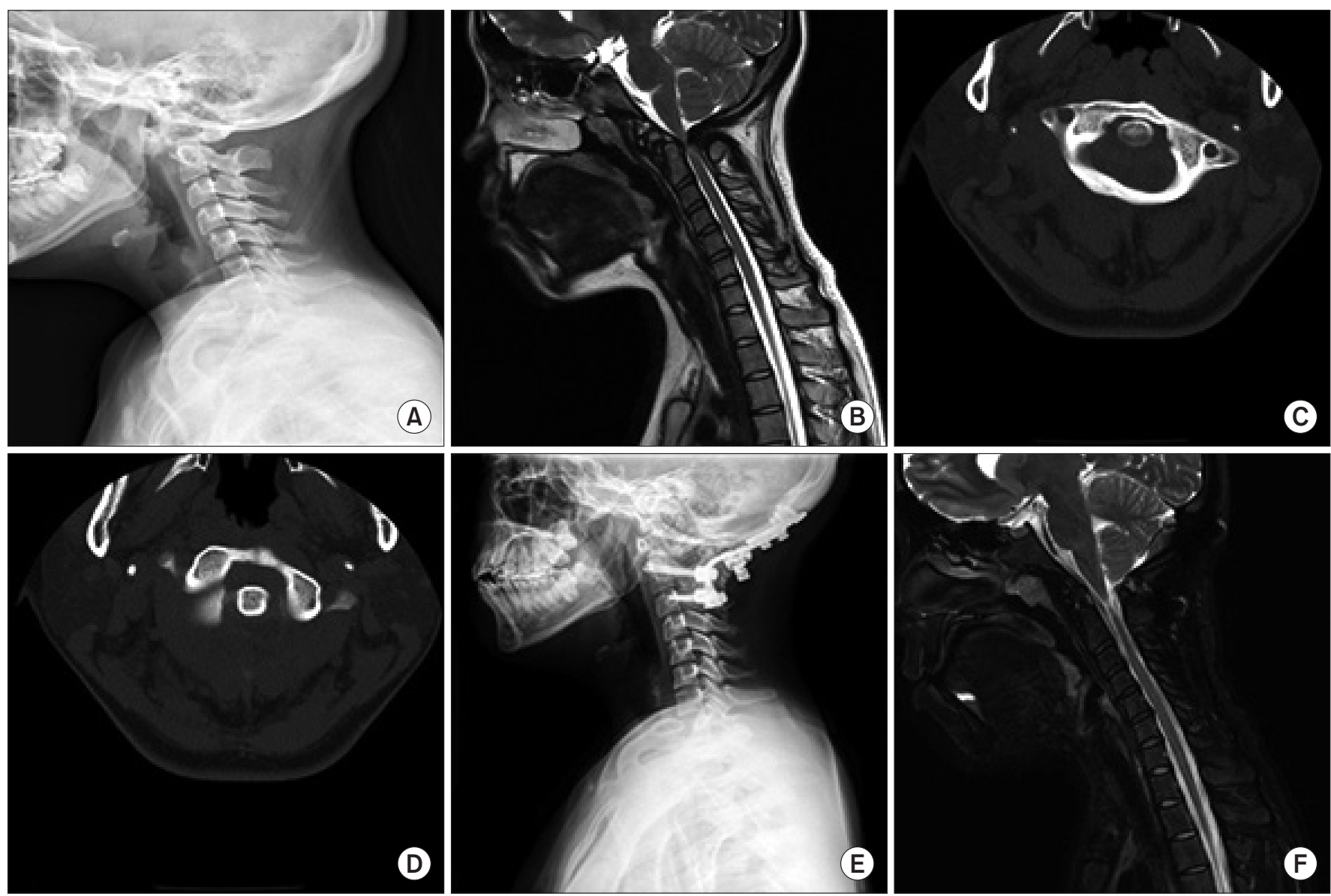

Fig. 1. The radiologic findings of the initial and postoperative state. (A) Lateral view of cervical spine X-ray. The fracture of odontoid process was demonstrated. (B) The initial magnetic resonance imaging (MRI) of cervical spine. MRI showed narrowing cervical canal, fracture of odontoid process, and cervical myelopathy at C1/C2. (C, D) Axial view of initial computed tomography (CT) of cervical spine. CT demonstrated old odontoid process fracture, anterior displacement of fractured odontoid process and C1. (E) Lateral view of cervical spine X-ray at postoperative state. Laminectomy and posterior metallic fixation at C1-3 were shown. (F) The follow-up MRI of cervical spine at postoperative state. MRI showed marked thinning with increased signal intensity in spinal cord at C2 level, myelomalacia with atrophic change of spinal cord. 